\title{
Clostridium difficile infection: review
}

\author{
Jacek Czepiel ${ }^{1}$ (D) - Mirosław Dróżdż ${ }^{2}$. Hanna Pituch ${ }^{3}$ - Ed J. Kuijper ${ }^{4,5} \cdot$ William Perucki $^{6}$ - Aleksandra Mielimonka ${ }^{7}$. \\ Sarah Goldman ${ }^{7}$ - Dorota Wultańska ${ }^{3}$. Aleksander Garlicki ${ }^{1} \cdot$ Grażyna Biesiada $^{1}$
}

Received: 27 January 2019 / Accepted: 12 March 2019 / Published online: 3 April 2019

(C) The Author(s) 2019

\begin{abstract}
Clostridium difficile ( $C$. difficile) is a Gram-positive, spore-forming, anaerobic bacillus, which is widely distributed in the intestinal tract of humans and animals and in the environment. In the last decade, the frequency and severity of $C$. difficile infection has been increasing worldwide to become one of the most common hospital-acquired infections. Transmission of this pathogen occurs by the fecal-oral route and the most important risk factors include antibiotic therapy, old age, and hospital or nursing home stay. The clinical picture is diverse and ranges from asymptomatic carrier status, through various degrees of diarrhea, to the most severe, life threatening colitis resulting with death. Diagnosis is based on direct detection of $C$. difficile toxins in feces, most commonly with the use of EIA assay, but no single test is suitable as a stand-alone test confirming CDI. Antibiotics of choice are vancomycin, fidaxomicin, and metronidazole, though metronidazole is considered as inferior. The goal of this review is to update physicians on current scientific knowledge of $C$. difficile infection, focusing also on fecal microbiota transplantation which is a promising therapy.
\end{abstract}

Keywords Antibiotic-associated diarrhea $\cdot$ Clostridium difficile $\cdot$ Diagnosis $\cdot$ Fecal transplantation $\cdot$ Treatment

\section{Introduction}

\section{CDI has become one of the most significant nosocomial infection}

Clostridium difficile (C. difficile) is a Gram-positive, anaerobic, spore-forming, toxin-producing bacillus, which was officially renamed in 2016 to Clostridioides difficile. New name reflects the taxonomic differences between this species and other members of the Clostridium genus [1, 2]. Spores of $C$. difficile are transmitted by the fecal-oral route, and the pathogen is widely present in the environment. Potential reservoirs for $C$. difficile include asymptomatic carriers, infected patients, the

Jacek Czepiel

jacek.czepiel@uj.edu.pl

Grażyna Biesiada

gbiesiada@op.pl

1 Department of Infectious and Tropical Diseases, Jagiellonian University, Medical College, Śniadeckich 5, 31-501 Krakow, Poland

2 Saint Ann's Hospital, Miechów, Poland

3 Department of Medical Microbiology, Medical University of Warsaw, Warsaw, Poland contaminated environment and animal intestinal tract (canine, feline, porcine, avian). Approximately 5\% of adults and 15-70\% of infants are colonized by $C$. difficile, and the colonization prevalence is several times higher in hospitalized patients or nursing home residents [3]. C. difficile was first isolated from a healthy newborn's stool in 1935 by Hall and O'Toole [4]. Until the 1970s, it was considered as a microorganism that is rarely, but present in normal intestinal microbiota. After the introduction of antibiotics, the role of C. difficile in the pathogenesis of large intestine diseases increased. In 1974, Tedesco et al. found that $21 \%$ of patients treated with clindamycin developed diarrhea. Pseudomembranes were found in $50 \%$ of cases, as

4 Department of Medical Microbiology, Centre for Infectious Diseases, Leiden University Medical Center, Leiden, The Netherlands

5 Centre for Infectious Diseases Research, Diagnostics and Laboratory Surveillance, RIVM, Bilthoven, The Netherlands

6 Department of Medicine, John Dempsey Hospital, University of Connecticut, Farmington, CT, USA

7 University Hospital, Krakow, Poland 
revealed by further endoscopic examination [5]. At the end of the twentieth century, the incidence of Clostridium difficile infection (CDI) markedly increased. Currently, CDI has become one of the most significant nosocomial infections, which affects all hospital wards.

\section{Risk factors associated with CDI}

\section{Antibiotic exposure, older age, and hospitalization are key factors for CDI development}

Significant patient-related risk factors for CDI are antibiotic exposure, older age, and hospitalization. Nearly every antibiotic has been associated with the development of CDI, including the drugs used for treatment of CDI: metronidazole and vancomycin. Broad spectrum penicillins and cephalosporins, clindamycin, and fluoroquinolones possess a higher risk for CDI induction than other antibiotics [3]. The risk for development of CDI is 8 - to 10 -fold higher during antimicrobial therapy and 4 weeks thereafter, and 3 -fold higher in the next 2 months [6]. Patient age $>65$ years increases the risk for CDI 5 to 10 -fold, compared with patients $<65$ years of age. Nonetheless, a significant proportion of CDI occurs in a younger population. Age $>65$ years is a significant risk factor not only for CDI itself, but also for poor clinical outcome including severity and mortality [3,7]. Although most cases of CDI are linked to healthcare exposure, either hospitalization or nursing home stay, recent studies suggest that the incidence of community-acquired CDI is growing, and might have recently reached up to $30 \%$ of all CDI cases [8]. The percentage of hospitalized patients with C. difficile colonization differs by country, patient age group, and length of hospitalization. During the first days of hospitalization, the incidence of $C$. difficile colonization ranges from 2.1 to $20 \%$ [9-13], and increases with longer hospital stay, e.g., from 20 to $45.4 \%$ in a study by Huang et al., from 2.1 to $50 \%$ after 1 month of hospitalization in a study by Clabots et al., and from 1 to $50 \%$ after $>1$ month of hospitalization in a study by Johnson et al. [12, 14, 15]. It must be noted that colonization does not necessarily mean symptomatic infection; it is suggested that only $25-30 \%$ of asymptomatic colonized patients develop diarrhea. $C$. difficile spores survive in the environment for several months [16]. Toilets, clinic furnishings, phones, and medical devices (thermometers, stethoscopes) may all serve as reservoirs for the $C$. difficile spores. The spores can be transferred to patients via the hands of healthcare personnel; therefore, good hand hygiene with soap and water and regular vinyl glove use is crucial to interrupt the transmission, as demonstrated by Johnson et al. [17]. Nursing home residents are at higher risk for
CDI than the overall population, but lower than hospitalized patients $(15 \%)$. This is mainly due to older age, comorbidities, more frequent hospitalizations, and more frequent antibiotic therapy in this group compared to the non-institutionalized population. $C$. difficile is the most common cause of nosocomial diarrhea [18]. It has been postulated that gastric acid suppression may have an influence on CDI development, but subsequent analysis adjusted for other comorbidities did not confirm this hypothesis $[19,20]$. This is in line with the observations that gastric acid did not kill the $C$. difficile spores [3]. Nonetheless, this topic remains controversial, as several studies and meta-analyses have found a significant association [21-23], whereas other have failed to associate proton pump inhibitors use with risk of CDI development [24-26].

Other well-defined risk factors for CDI include inflammatory bowel disease, gastrointestinal surgeries, immunological incompetence caused by malignant neoplasms, transplantations, chronic kidney diseases, or immunosuppressant use $[3,27]$.

\section{Pathogenesis}

\section{The main protective barrier against CDI is the normal intestinal microflora}

Infection with $C$. difficile mostly occurs as a result of spore transmission. Spores are resistant to heat, acid, and antibiotics. The main protective barrier against CDI is the normal intestinal microflora. After reaching the intestine, bile acids play an important role in the induction of $C$. difficile spore germination [28]. Bile acids are cholesterol derivatives produced and transformed in the liver. They facilitate the absorption of fats and fat soluble vitamins in the intestine. They support digestion, improve gastrointestinal motility, and actively affect bacterial flora $[29,30]$. We distinguish between primary and secondary bile acids. Primary bile acids, cholic acid and chenodeoxycholic acid, are synthesized in the liver from cholesterol and are secreted into the intestine after eating. Then, as a result of active transport, about $95 \%$ of primary bile acids are reabsorbed in the final part of the intestine. The remaining, non-reabsorbed bile acids may undergo 7a-dehydroxylation by gut bacteria, transforming into secondary bile acids, deoxycholic acid and lithocholic acid [30]. In vitro, primary bile acids generally stimulate germination of $C$. difficile spores; the secondary bacteria inhibit this process $[31,32]$. Moreover, in patients with CDI, there are changes in the fecal content of bile acids. Allegretti et al. showed a higher concentration of secondary bile acids in the feces of healthy people compared to CDI, while primary bile acid concentration was higher in patients with recurrent CDI compared to patients 
with their first episode of infection [33]. However, it has to be reinforced that the influence of bile acids is likely more complex than the simple model where primary bile acids strictly promote and secondary bile acids inhibit $C$. difficile germination and vegetation as described in the excellent review by Baktash et al. [29].

When the balance of gut microorganisms is disrupted, C. difficile starts to dominate and colonize the large intestine which might be the first step of infection. As mentioned previously, only a portion of colonized patients will develop symptoms of CDI [3]. The pathogen is not invasive, and virulence is mostly due to enzymes, such as collagenase, hyaluronidase, chondroitin-sulfatase, as well as toxins, which damage the epithelial cell cytoskeleton, leading to disruption of tight junctions, fluid secretion, neutrophil adhesion, and local inflammation. The result is a breakdown of gut barrier integrity and loss of functionality $[29,34]$. C. difficile produces two important in disease pathogenesis types of toxins, $\mathrm{A}$ and $\mathrm{B}$, which are both enterotoxic and cytotoxic; however, traditionally, toxin A is named "enterotoxin A" and toxin B, "cytotoxin B." C. difficile transferase (CDT; or binary toxin) is a third toxin produced by some $C$. difficile strains, including the epidemic PCR ribotypes 027 . It probably can form microtubule-based protrusions on epithelial cells, which theoretically could have a clinical impact. There are reports of severe CDI development caused by the $\mathrm{TcdA}^{-} \mathrm{TcdB}^{-} \mathrm{CDT}^{+}$ strain [35].

Toxins are transported to the cell cytoplasm, where they inactivate the Rho family of GTPases. The Rho protein takes part in actin polymerization, and therefore stabilizes the cell cytoskeleton. As a result of Rho protein inactivation, the inflammatory process intensifies. In more severe cases, microulcerations covered with pseudomembranes (composed of destroyed intestinal cells, neutrophils, and fibrin) start to occur on the intestinal mucosal surface. Initial studies on animal models suggested that toxin A plays a dominant role, and the action of toxin $\mathrm{B}$ may occur only via the tissue damage caused by toxin A [36]. However, in studies involving human colonic tissue, TcdB was a potent inflammatory toxin, whereas TcdA was even weaker, and both toxins were able to elicit CDI symptoms independently [36-38].

The $C$. difficile BI/NAP1/027 strain is hypervirulent and resistant to fluoroquinolones, exhibits intensive spore production, and is responsible for the most severe CDI cases. The C. difficile BI/NAP1/027 epidemic strain is characterized by two mutations in the toxin regulatory gene $t c d C$, an 18 basepair (bp) deletion, and deletion at position 117, which leads to increased production of toxins A and B [3, 39]. It was first isolated at the beginning of the twenty-first century in North America and Europe. BI/NAP1/027 was extremely rare before 2000; in the first two large epidemics of CDI in North America at the beginning of the last decade, the percentage of CDI caused by BI/NAP1/027 was $51 \%$ in the US and $84 \%$ in Canada [39, 40]. Analyzing the data of 6000 CDI cases prior to 2001, only 14 cases attributed to BI/NAP1/027 were identified, representing only $0.2 \%$ of all cases [40]. Furthermore, numerous cytokines play a role in CDI pathogenesis, including IL- 8 , IL- $1 \beta$, IL- 6 , TNF $\alpha$, INF $\gamma$, and leukotriene B4 [41-43].

\section{Clinical manifestation}

\section{CDI clinical picture can vary from the asymptomatic carrier state to life-threatening colitis resulting with death}

The clinical picture of CDI is very heterogenous, and ranges from the asymptomatic carrier state, mild or moderate diarrhea, to life-threatening fulminant colitis. Although the incubation period is not precisely defined, and some reports suggest 2-3 days, more recent studies demonstrate that the incubation period might be even longer than 3 days and is very individual-dependent [44-47]. CDI can affect every part of the colon, but the distal segment is most commonly infiltrated. Most patients with CDI suffer from mild diarrhea and experience recovery spontaneously after 5-10 days of antibiotic therapy withdrawing. Diarrhea occurs in most cases during, or directly after antimicrobial therapy, although CDI onset might be also a couple of weeks afterwards. The clinical features of CDI, in addition to watery diarrhea, include abdominal pain, fever, nausea and vomiting, weakness, and loss of appetite. Fecal occult blood test is often positive, although active bleeding is rarely present [47]. In the most severe clinical presentation of CDI, symptoms are life-threatening, and include significant dehydration, abdominal distension, hypoalbuminemia with peripheral edema, and subsequent circulatory shock. Other severe complications of CDI include toxic megacolon, colon perforation, intestinal paralysis, kidney failure, systemic inflammatory response syndrome, septicemia, and death [47]. Extracolonic manifestations of CDI are rare, and most commonly involve small intestine infiltration, reactive arthritis, and bacteremia [43]. Mortality rate directly due to $\mathrm{CDI}$ is estimated at $5 \%$, whereas mortality associated with CDI complications reaches $15-25 \%$, and up to $34 \%$ in intensive care units (ICU). Mortality doubles in ICU patients with CDI, as compared with ICU patients without CDI [7, 48, 49]. Poor outcome is associated with older age, high leukocytosis, hypoalbuminemia, and high creatinine level $[43,50]$. It has been also shown that first-ever CDI episode increases the overall risk of death [7].

CDI relapse of symptoms occur most commonly during the first week after the initial episode when treatment is complete. After effective treatment of first CDI episode, at least one new recurrent episode occurs in $10-25 \%$ of patients, and up to $65 \%$ in patients who experienced already 
$>1$ recurrent CDI $[51,52]$. There is evidence to show that half of the recurrent CDI cases are due to relapses of infection with the original strain, whereas the other half is caused by re-infection with different strains. Impaired immune response to $C$. difficile toxins, as well as new exposure to spores, is thought to contribute to recurrences. Antibiotic resistance does not seem to influence the risk of recurrences [18, 43, 53].

\section{Prevention}

\section{Prevention strategies should be implemented in every suspected case, not only in confirmed patients}

Strategies for prevention of CDI include the use of gloves and disposable gowns by healthcare personnel and visitors during the whole diarrheal episode. After every direct contact with a CDI-patient, everyone should wash their hands with soap and water. Alcohol-based hand hygiene products do not damage the $C$. difficile spores, whereas the mechanical hand washing with the use of running water and soap prevents spread of the spore. Optimally, every patient with CDI should be isolated in a single room. If this is not possible, contact between patients should be avoided, (e.g., reading the same books/magazines, using the same phone), and the patient should have his or her own furnishing. There are no current recommendations to screen asymptomatic carriers, as effectiveness has not been proven. Chlorine-based solutions are commonly recommended for environmental cleaning, with $1000 \mathrm{ppm}$ of chlorine concentration being effective, and $5000 \mathrm{ppm}$ being the most optimal choice [54]. Prevention strategies should be implemented in every suspected case, not only in confirmed patients. After discharge, the patient's room should be carefully decontaminated [3].

\section{Diagnosis}

\section{No single test is suitable as a stand-alone test confirming CDI}

CDI should first be considered when diarrhea symptoms are present ( $\geq 3$ loose stools during $24 \mathrm{~h}$ ). The diagnosis of CDI is based on detection of $C$. difficile toxins directly in a stool sample, most commonly with an enzyme immunoassay (EIA), which provides rapid turnaround time (about 1-2 h), as well as sensitivity of $75-85 \%$ and specificity of $95-100 \%$. Because of its low cost and ease of use, this is the most popular test in all laboratories. Tests detecting $C$. difficile antigens are based on the detection of glutamate dehydrogenase (GDH) and are characterized by ease of use and rapid turnaround time as well as a specificity of almost $100 \%$. However, they do not distinguish whether the strain is toxigenic (specificity of 59\%) $[18,55]$. It should be pointed out that old-generation assays (using latex agglutination) had sensitivity of $58-68 \%$ and specificity of $89-99 \%$. In 2009, tests that use amplification of nucleic acid (NAAT, nucleic acid amplification test) were introduced. They are based on either a PCR method or isothermal amplification. NAAT have higher sensitivity (80$100 \%$ ) and specificity (87-99\%) compared to an EIA test. The specificity is especially high, reaching $95 \%$, when a negative result is obtained. In this situation, another cause of diarrhea should be considered $[47,56,57]$. The NAAT have also limitations, namely, high cost and some interpretation difficulties. PCR detects the presence of a toxin encoding gene, thus confirms the presence of $C$. difficile toxin-producing strain, but it does not necessarily mean that the strain produces any toxins at the moment. If the diarrhea is of other origin, detection of such strain would become misleading, as it would pursue further treatment towards CDI. Persistent and often ineffective treatment of only colonized patients does not improve their clinical situation. When dealing with such diagnostic difficulties, comprehensive diagnostic evaluation of other potential diarrhea causing disorders is required. A cytotoxic assay test (CYTA) is not routinely used in microbial culture due to its slow turnaround time and lack of standardization (48-72 h) [3, 47].

According European Society of Clinical Microbiology and Infectious Diseases (ESCMID) guidance, no single test is suitable as a stand-alone test confirming CDI. The best way to optimize diagnosis of CDI is to combine two tests in algorithm. The first test should be a test with high negative predictive value (it can either be a GDH EIA or NAAT). The second test should be a test with a high positive predictive value (it is toxin $\mathrm{A} / \mathrm{B}$ EIAs). If the first test is negative, it excludes CDI. If the first test is positive, the second test (toxin $\mathrm{A} / \mathrm{B}$ EIAs) should be performed. If the second test is positive, it confirms CDI. If the second test is negative, the case needs to be clinically evaluated, and such result can be seen in three situations: CDI with toxin levels below the threshold of detection, false-negative toxin A/ B EIA result, or $C$. difficile carriage. Samples with a negative GDH result but that are positive for toxin need to be retested, as this is an invalid result [58]. Flow chart of CDI diagnosis is presented on Fig. 1.

Proper management in the pre-analytical phase is extremely important, as the toxin present in a stool sample is easily degraded at room temperature, and after about $2 \mathrm{~h}$, it can be no longer detected in the acquired material. Once the stool sample is obtained, it should be stored at refrigerator temperature $\left(+4{ }^{\circ} \mathrm{C}\right)$ and used for testing within the next $24 \mathrm{~h}$ [59]. The test is only performed on diarrheal sample unless ileus is suspected-in such case, it is acceptable to obtain a sample 


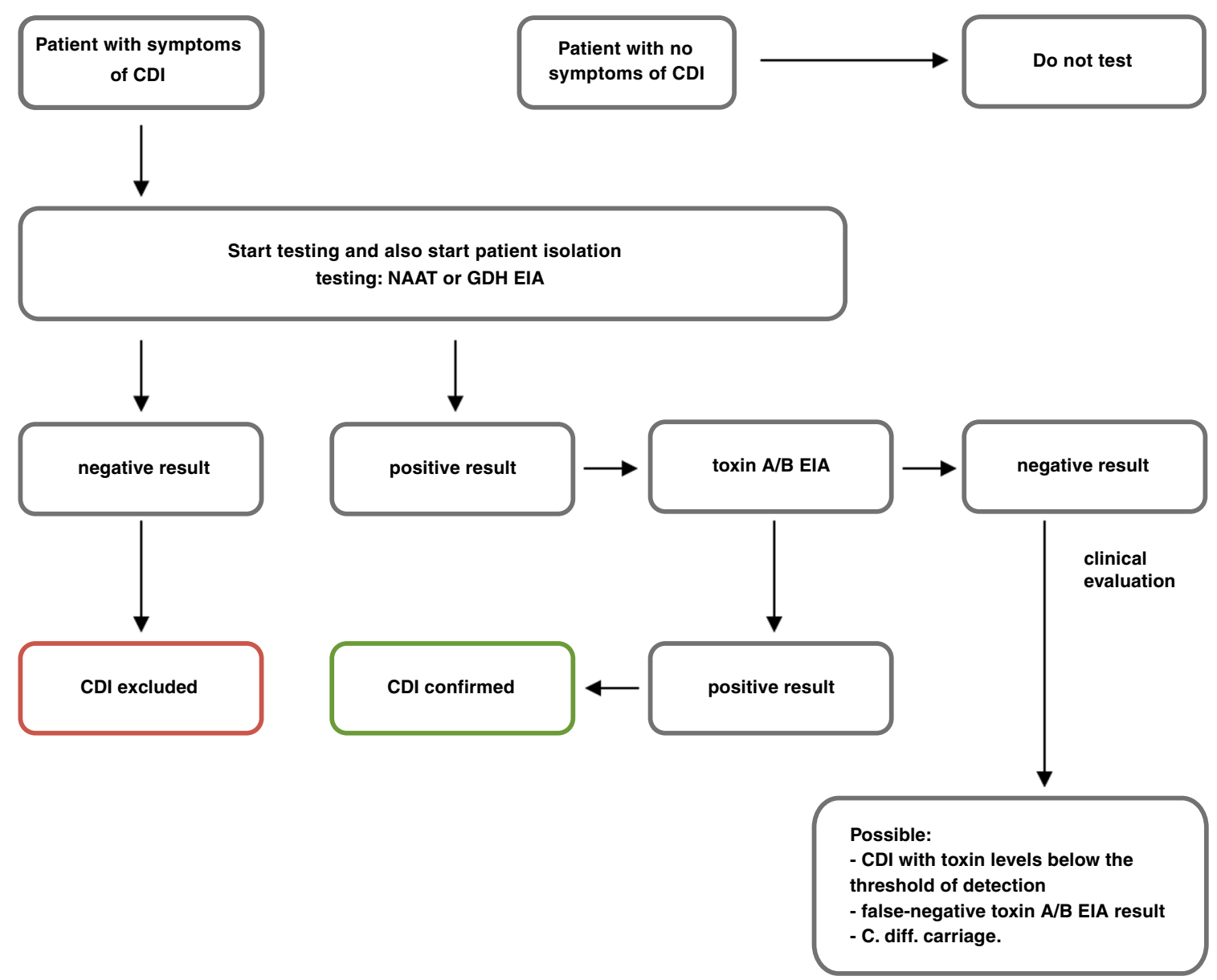

Fig. 1 Flow chart of CDI diagnosis

by rectal swab. With the exception of epidemiological purposes, it is not recommended to test stool samples obtained from asymptomatic patients. It is also not recommended to repeat testing for $C$. difficile after successful treatment is completed as there is a significant proportion of patients who test positive and their treatment does not need to be continued or repeated [18, 54, 60].

Endoscopic evaluation is also useful; however, it should be emphasized that it is not performed in patients with uncomplicated CDI that was confirmed with immunological tests. Endoscopy is indicated if diagnostic problems occur, namely, a typical CDI presentation with negative $C$. difficile test results, no response to standard course of antibiotics or when an alternative diagnosis is suspected, and direct visualization and/or biopsy of the bowel mucosa is needed. If the colonoscopy is performed, limited flexible sigmoidoscopy is preferred with minimal or no air insufflation to avoid perforation of the inflamed colon. The pseudomembranes found during the procedure are elevated, white to yellow lesions, typically about $2 \mathrm{~cm}$ in diameter, which are irregularly distributed and separated by normal mucosa. They are not removed by intestinal wall rinsing. The distribution of pseudomembranes tends to vary. Not all patients with CDI have pseudomembranes, and their absence does not rule out $C$. difficile infection. For example, pseudomembranes are rarely in recurrent CDI or in CDI among patients with inflammatory bowel disease [61-63]. On the other hand, pseudomembranous colitis can be caused by a number of different etiologies, like Behcet's disease, collagenous colitis, inflammatory bowel disease, ischemic colitis, and also other infections, like CMV or enterohemorrhagic Escherichia coli O157:H7 [64].

Abdominal imaging (X-ray, ultrasound) in patients with CDI reveals distended bowel loops, often with wall thickening. Their use is of the highest importance when diagnosing CDI complications. Ultrasound imaging is an especially good method of monitoring the width of colon [18, 43]. Computed tomography of the abdomen and pelvis with oral and intravenous contrast is useful among patients with severe CDI, helping to evaluate for presence of toxic megacolon, bowel perforation, or other findings warranting surgical intervention [65]. 
Laboratory findings reveal high leukocytosis, elevated Creactive protein, and in the most severe cases hypoalbuminemia as well as acute kidney injury [43].

\section{CDI treatment}

\section{Vancomycin and fidaxomicin are the cornerstone of CDI treatment}

Treatment should only be started in patients with CDI symptoms; presence of the $C$. difficile toxin without symptoms of the infection is not an indication for treatment. In 2014, the ESCMID guidelines were published in which two drugs metronidazole and vancomycin were the cornerstone of CDI treatment. Metronidazole was first-line drug in non-severe CDI, while vancomycin was the drug of choice for severe CDI [66]. Since then, the results of two identical, phase 3, multicenter, randomized, double dummy, double-blind, active-controlled, parallel-design efficacy studies (RCT) showed superiority of vancomycin relative to metronidazole. Clinical success occurred in $210(81 \%)$ of 259 patients treated with vancomycin versus $202(73 \%)$ of 278 patients who were treated with metronidazole $(p=0.02)$. However, among patients with severe disease, a statistically significant relationship was not achieved (clinical success achieved $78.5 \%$ in the vancomycin group compared with $66.3 \%$ in the metronidazole group $(p=$ 0.059 ) [67]. In 2017, meta-analysis by Nelson et al. also concluded that metronidazole is inferior compared to vancomycin in the treatment of CDI [68].

Fidaxomicin is a drug that has been available since 2011. It is a macrocyclic, bactericidal antibiotic of narrow spectrum, directed primarily against Gram-positive pathogens. It has high efficacy against $C$. difficile, with no significant influence on the physiological flora of the colon. Fidaxomicin has efficacy comparable to vancomycin and in some groups higher effectiveness in reducing CDI recurrence. CDI recurrence after treatment of the first episode with fidaxomicin occurred in $15 \%$ of patients compared to $25 \%$ of patients treated with vancomycin. However, the same reduction in recurrence of the BI/NAP/027 strain was not observed [69, 70]. Fidaxomicin is also associated with lower percentage of CDI recurrence than vancomycin ( $20 \%$ vs $36 \%$ ) in patients, who experienced CDI recurrence $>4$ weeks after the treatment of the previous episode [71]. Another published meta-analysis suggested that fidaxomicin may be considered as first-line therapy for CDI [72]. Moreover, Guery et al. in 2017 showed that a tapered fidaxomicin treatment (days 1-5, $200 \mathrm{mg}$ two times a day, followed by once daily on alternating days during days $7-25)$ is superior $(p=0.03)$ than vancomycin $(125 \mathrm{mg}$ oral capsules, four times daily on days 1-10) in resulting in a sustained clinical cure (30 days after end of treatment) in CDI. Recurrence rate at 90 day was also lower among fidaxomicin arm then vancomycin arm (9\% vs $18 \%$, respectively, $p=$ $0,048)$ [73].

In 2017, Infectious Diseases Society of America (IDSA) and Society for Healthcare Epidemiology of America (SHEA) updated their guidelines, pointing that vancomycin and fidaxomicin are the cornerstone of CDI treatment [47] (Table 1).

There are no uniform criteria stratifying non-severe and severe CDI. Based on the commonly known risk factors for CDI severity and CDI criteria used by other researchers, we have proposed to define the severity of CDI as follows.

Severe CDI presents with or develops two or more of the following severity markers during the course of the disease: hypoalbuminemia (serum albumin $<3 \mathrm{~g} / \mathrm{dl}$ ), white blood cell count $\geq 15,000$ cells $/ \mathrm{mm}^{3}$, creatinine $>1.5 \times$ baseline (or glomerular infiltration rate reduced by $25 \%$ from baseline), or temperature $>38.5{ }^{\circ} \mathrm{C}$. Fulminant (severe complicated CDI), defined as CDI that presents with or develops at least one of the following signs or symptoms: admission to an intensive care unit, hypotension with or without use of vasopressors, ileus, toxic megacolon, mental status changes, serum lactate levels $>2.2 \mathrm{mmol} / \mathrm{l}$, or any evidence of end organ failure [74].

If there is high suspicion of CDI with a negative ELISA assay, it is reasonable to start empiric antibiotic therapy for CDI. Other antibiotics that show activity against $C$. difficile include teicoplanin, tigecycline, bacitracin, and nitazoxanide. However, they are not included in CDI treatment recommendations and they may be considered when basic therapeutic options have run out. Pregnant and breast-feeding women should be treated with orally administered vancomycin in typical doses [3, 75-77]. The use of additional antibiotics (other than those treating CDI) is associated with increased risk of prolonged diarrhea and CDI recurrence, which is why they should be discontinued. However, if such therapy is indispensable, it should be continued preferably with antibiotics that are associated with lower risk of CDI, such as macrolides, aminoglycosides, sulfonamides, vancomycin, or tetracyclines. Some authors suggest that in this situation, prolonged treatment with antibiotics acting against $C$. difficile should be discontinued a week after the other broad-spectrum therapy is completed [78, 79]. According to authors of this paper, such regimen is worth implementing, if the additional CDI treatment does not exceed 7 to 10 days.

The role of probiotics in the treatment and prevention of CDI is completely unknown. Three large trials, including a meta-analysis have shown a positive effect of probiotics in the prevention of primary CDI. However, it should be emphasized that the meta-analysis has a variety of limitations. The studies included in the meta-analysis differ in regard to doses and types of probiotics, $C$. difficile strains, doses and types of antibiotics, time of therapy, and ultimately in some of these studies the number of CDI was very low in all study arms. Considering the pathophysiology of CDI, it appears that 
Table 1 Antibiotic regimens used in the treatment of $C$. difficile infection $[29,47,66,67,69-73]$
First episode of the infection

Non-severe disease

Severe disease

Fulminant disease (previously referred as severe complicated)
- Vancomycin $125 \mathrm{mg}$ orally four times a day for 10 days

OR

- Fidaxomicin $200 \mathrm{mg}$ orally twice a day for 10 days

- If above agents are unavailable: metronidazole $500 \mathrm{mg}$ orally three times a day for 10 days

- Vancomycin $125 \mathrm{mg}$ orally four times a day for 10 days

OR

- Fidaxomicin $200 \mathrm{mg}$ orally twice a day for 10 days

- Vancomycin $500 \mathrm{mg}$ orally or via nasogastric tube four times a day

AND

- Metronidazole $500 \mathrm{mg}$ IV 3 times a day + alternatively

If ileus is present: vancomycin per rectum (vancomycin $500 \mathrm{mg}$ in $100 \mathrm{ml}$ saline as enema) four times a day* (10-14 days)

First recurrence

If the first episode was treated with metronidazole or fidaxomicin:

- Vancomycin $125 \mathrm{mg}$ orally four times a day for 10 days

If the first episode was treated with vancomycin:

- Vancomycin pulsed-tapered orally (each dose $125 \mathrm{mg}$ ):

\# Four times daily for 10-14 days and then

\# Twice a day for 7 days, than

\# Once a day for 7 days, than

\# Every 2 or 3 days for $2-8$ weeks

OR

- Fidaxomicin $200 \mathrm{mg}$ orally twice a day for 10 days

Second of subsequent recurrences

- Vancomycin pulsed-tapered orally (regimen as above)

OR

- Fidaxomicin $200 \mathrm{mg}$ orally twice a day for 10 days;

OR

- Vancomycin $125 \mathrm{mg}$ orally four times a day for 10 days, followed by rifaximin $400 \mathrm{mg}$ three times daily for 20 days

OR

- Fecal microbiota transplantation

*If there is partial ileus, vancomycin should be administered both orally and rectally; if the ileus is complete, only rectal vancomycin should be used. Rectal vancomycin administration is associated with a risk of large bowel perforation, and should only be used in those patients who do not respond to oral therapy; some patients may have delayed response to treatment and clinicians should consider extending treatment duration from 10 to 14 days in such situations

probiotics may be a part of CDI prevention or treatment; however, we still lack properly randomized studies addressing this problem [50, 80-84]. There are insufficient data at this time to recommend administration of probiotics for primary, secondary prevention or treatment of CDI $[47,66]$.

Asymptomatic $C$. difficile carriers have high concentrations of antibodies directed against toxins A and B [85]. Based on this knowledge, there are studies on intravenous administration of immunoglobulins as well as monoclonal antibodies that may be useful in both the treatment and prevention of CDI recurrences. Successful immune therapy for the treatment or prevention of CDI would be an interesting way of fighting the disease, as in contrast to vancomycin, metronidazole, and fidaxomicin, it would not disrupt the bacterial flora of the host [86]. Anti-toxin antibodies are of great importance in the development of immunity against CDI. It has been shown that naturally produced anti-toxin antibodies in $C$. difficile colonized patients who did not develop diarrhea have protective effects [87, 88]. The administration of monoclonal antibodies directed against these toxins markedly reduced the risk of CDI recurrence. Thus, there is a chance that in the near future, we will have new forms of protection against $C$. difficile recurrence which might even be used when fighting the first infection [89]. Bezlotoxumab (a monoclonal antibody that binds to 
C. difficile toxin B) was approved by the FDA in 2016 for prevention of recurrent $\mathrm{CDI}$ in patients with high risk of CDI recurrence. The registration trial, which included over 2500 patients, showed that bezlotoxumab together with standard oral antibiotic therapy was associated with a significantly lower rate of recurrent infection than oral antibiotic therapy alone (17 versus $28 \%$ ). At the same time, no similar effect was shown for actoxumab (a monoclonal antibody that binds to $C$. difficile toxin A). Bezlotoxumab is undoubtedly a significant achievement in CDI prevention; however, its use is limited by high cost and potential side effects. In the group of patients treated with bezlotoxumab, the incidence of acute decompensated heart failure was significantly higher when compared with the placebo group ( $12.7 \%$ vs $4.8 \%$ respectively) [90].

\section{Fecal microbiota transplantation}

\section{Fecal microbiota transplantation have the highest rate of prevention of recurrent CDI among all therapeutic options}

The fecal microbiota transplantation (FMT) procedure has been known for over 1000 years, and was first described by a traditional Chinese medicine doctor Ge Hong, who lived during the Dong Lin Dynasty period (284-364 BC). He applied human fecal suspension orally to patients with severe diarrhea or food poisoning [91]. In Europe, the idea was first used in veterinary medicine by the Italian anatomist Fabricius Aquapendente in the seventeenth century [92]. Modern medicine first performed fecal transplantation in 1958. Eiseman et al. used fecal enema as therapy for pseudomembranous enterocolitis $(C$. difficile had not been routinely identified at that time) [93]. The first report about fecal transplantation in patient with confirmed C. difficile infection was published in 1983 [94].

Nowadays, abnormal gut microbiota is considered a key factor in CDI development. Relatively short antimicrobial therapy might dramatically reduce the amount of intestinal microbiota, but recovery may last several months. During that specific period, patients lack their protective barrier, and with exposure to spores an infection might develop quickly. Even though the gut microbiota is composed of thousands of species of microbes, it is thought that Bacteroides and Firmicutes play predominant role in immunological responses against C. difficile [95].

Antibiotic withdrawal together with FMT have the highest rate of prevention of recurrent CDI among all therapeutic options [96-98]. Nood et al. performed openlabel, randomized, controlled trial, which compared three treatment regimens: the infusion of donor feces preceded by an abbreviated regimen of vancomycin and bowel lavage, a standard vancomycin regimen, and a standard vancomycin regimen with bowel lavage. The study was stopped after an interim analysis. Eighty-one percent of the patients in FMT group had resolution of CDI comparing with only $31 \%$ patients receiving vancomycin alone and $23 \%$ receiving vancomycin with bowel lavage ( $p<0.001$ comparing with both control group). Moreover, no significant differences in adverse events among the three study groups were observed except for mild diarrhea and abdominal cramping in the FMT group on the infusion day. After FMT, patients showed increased fecal bacterial diversity, similar to that in healthy donors [98]. One hundred percent effectiveness was observed in the 27-patient study by Dutta et al. where fecal material was directly introduced into small and large intestine [96]. Louie et al. performed interesting experiments with fecal microbes contained in gelatin capsules. According to the protocol, 27 patients received 24-34 capsules, and the effectiveness of the therapy was also $100 \%$ [97].

Transplantation with frozen fecal material, first described by Borody and Khoruts, simplified the procedure [99]. Stool samples can be stored at $-80{ }^{\circ} \mathrm{C}$ and used during next 5-6 months. Some stool banks extend storing period for 2 years [100]. FMT procedure has not yet been standardized. The donated stool is mixed with normal saline solution, homogenized, and filtrated to separate the solid parts, to obtain fluid material. Fecal transplant can be administrated via oral capsules, lower gastrointestinal (GI) tract procedure (colonoscopy, retention enema), or upper GI tract procedure (nasojejunal/nasoduodenal tube) [101]. Some potential complications of FMT are connected with delivery method (e.g., perforation with colonoscopy, aspiration pneumonia with upper GI administration). However, the frequency of complications associated with FMT is likely similar to the frequency of complications when these procedures are performed for other indications. It seems that the effectiveness of FMT is higher after lower GI tract administration when compared to upper GI tract administration [101]. Retention enema is a procedure with a low cost, easily accessible, and with relatively low risk of complications; however, it may be difficult to maintain stool transplant and in such a situation, patients require repeated enema [101]. FMT is highly promising treatment of CDI, and several large "feces banks" have been developed. In the Netherlands, treatment with FMT is organized at a national level by the "Netherlands Donor Feces Bank" (NDFB, https://www.ndfb.nl/) at Leiden University Medical Center. Since 2016, NDFB has received more than 120 requests for treatment of FMT for patients with recurrent CDI. Each request is discussed by a panel of experts comprising of medical microbiologists, gastroenterologists, and infectious disease physicians. Of all received requests, only $80 \%$ 
fulfilled to the criterium of recurrent CDI and were treated. The success rate was very high, more than $90 \%$, and clearly associated with the stringent inclusion criteria (E.T Terveer,. K.E Vendrik and E.J.Kuijper, manuscript in preparation). Two main concerns are the risk of transferring infectious pathogens from the donor to the recipient, and development of autoimmunological disorders. Although the possibility of infecting the recipient with fecal material still exists, it is minimalized by proper donor screening. Potential donors should be healthy, have daily formed bowel movement, and screened for bacterial, viral, and parasites infection, as it is presented in thorough Terveer et al. study [100]. The influence of gut microbiota on some immune-mediated diseases such as irritable bowel disease raises concerns about long-term side effects of fecal transplantation. This field merits further investigation in the future [86].

\section{Conclusion}

In the past decade, CDI became one of the most detrimental nosocomial infections. It is of most importance to remember that CDI prevention starts with healthcare professional education regarding such preventive measures, as hand washing, gloves wearing, proper decontamination of medical devices and patient's environment, as well as optimal antibiotic management. Hospitalized elderly patients treated with antibiotics are at the highest risk for CDI. FMT is highly promising treatment of CDI.

\section{Compliance with ethical standards}

Conflict of interest The authors declare that they have no conflict of interest.

\begin{abstract}
Abbreviations $C D I$, Clostridium difficile infection; $C D T$, binary toxin; CYTA, cytotoxic assay test; $C$. difficile, Clostridium difficile; EIA, enzyme immunoassay; $F M T$, fecal microbiota transplantation; $G D H$, glutamate dehydrogenase; GI, gastrointestinal; $I C U$, intensive care unit; $N A A T$, nucleic acid amplification test
\end{abstract}

Open Access This article is distributed under the terms of the Creative Commons Attribution 4.0 International License (http:// creativecommons.org/licenses/by/4.0/), which permits unrestricted use, distribution, and reproduction in any medium, provided you give appropriate credit to the original author(s) and the source, provide a link to the Creative Commons license, and indicate if changes were made.

\section{References}

1. Oren A, Garrity GM (2017) List of new names and new combinations previously effectively, but not validly, published. Int J Syst Evol Microbiol 67:3140-3143
2. Lawson PA, Citron DM, Tyrrell KL, Finegold SM (2016) Reclassification of Clostridium difficile as Clostridioides difficile (Hall and O'Toole 1935) Preevot 1938. Anaerobe 40:95-99

3. Leffler DA, Lamont JT (2015) Clostridium difficile infection. N Engl J Med 373:287-288

4. Hall IC, O'Toole E (1935) Intestinal flora in newborn infants with description of a new pathogenic anaerobe. Am J Dis Child 49:390-402

5. Tedesco FJ, Barton RW, Alpers DH (1974) Clindamycinassociated colitis. A prospective study. Ann Intern Med 81:429433

6. Hensgens MP, Goorhuis A, Dekkers OM, Kuijper EJ (2012) Time interval of increased risk for Clostridium difficile infection after exposure to antibiotics. J Antimicrob Chemother 67:742-748

7. Czepiel J, Kędzierska J, Biesiada G et al (2015) Epidemiology of Clostridium difficile infection: results of a hospital-based study in Krakow, Poland. Epidemiology \& Infection 143:3235-3243

8. Khanna S, Pardi DS, Aronson SL et al (2012) The epidemiology of community-acquired Clostridium difficile infection: a population based study. Am J Gastroenterol 107:89-95

9. Hung YP, Lin HJ, Wu TC et al (2013) Risk factors of fecal toxigenic or nontoxigenic Clostridium difficile colonization: impact of Toll-like receptor polymorphisms and prior antibiotic exposure. PLoS One 8:e69577

10. Loo VG, Bourgault AM, Poirier L et al (2011) Host and pathogen factors for Clostridium difficile infection and colonization. N Engl J Med 365:1693-1703

11. Eyre DW, Griffitths D, Vaughan A et al (2013) Asymptomatic Clostridium difficile colonisation and onward transmission. PLoS One 8:e78445

12. Clabots CR, Johnson S, Olson M et al (1992) Acquisition of Clostridium difficile by hospitalized patients: evidence for colonized new admissions as a source of infection. J Infect Dis 166: 561-567

13. Marciniak C, Chen D, Stein AC et al (2006) Prevalence of Clostridium difficile colonization at admission to rehabilitation. Arch Phys Med Rehabil 87:1086-1090

14. Johnson S, Clabots CR, Linn FV et al (1990) Nosocomial Clostridium difficile colonization and disease. Lancet 336:97-100

15. Hung YP, Lee LC, Lin HJ et al (2015) Clinical impact of Clostridium difficile colonization. J Microbiol Immunol Infect 48:241-248

16. Hensgens MPM, Keessen EC, Squire MM et al (2012) Clostridium difficile infection in the community: a zoonotic disease? Clin Microbiol Infect 18:635-645

17. Johnson S, Gerding DN, Olson MM et al (1990) Prospective, controlled study of vinyl glove use to interrupt Clostridium difficile nosocomial transmission. Am J Med 88:137-140

18. Simor AE (2010) Diagnosis, management, and prevention of Clostridium difficile infection in long-term care facilities: a review. J Am Geriatr Soc 58:1556-1564

19. Kyne L, Sougioultzis S, McFarland LV, Kelly CP (2002) Underlying disease severity as a major risk factor for nosocomial Clostridium difficile diarrhea. Infect Control Hosp Epidemiol 23: 653-659

20. Novack L, Kogan S, Gimpelevich L et al (2014) Acid suppression therapy does not predispose to Clostridium difficile infection: the case of the potential bias. PLoS One 9:e110790

21. Arriola V, Tischendorf J, Musuuza J et al (2016) Assessing the risk of hospital-acquired Clostridium Difficile infection with proton pump inhibitor use: a meta-analysis. Infect Control Hosp Epidemiol 37:1408-1417

22. Deshpande A, Pasupuleti V, Thota P et al (2015) Risk factors for recurrent Clostridium difficile infection: a systematic review and metaanalysis. Infect Control Hosp Epidemiol 36:452-460 
23. Trifan A, Stanciu C, Girleanu I et al (2017) Proton pump inhibitors therapy and risk of Clostridium difficile infection: systematic review and meta-analysis. World J Gastroenterol 23:6500-6515

24. Beaulieu M, Williamson D, Pichette G, Lachaine J (2007) Risk of Clostridium difficile-associated disease among patients receiving proton-pump inhibitors in a Quebec medical intensive care unit. Infect Control Hosp Epidemiol 28:1305-1307

25. Shah S, Lewis A, Leopold D et al (2000) Gastric acid suppression does not promote clostridial diarrhoea in the elderly. QJM 93:175181

26. Campbell KA, Phillips MS, Stachel A et al (2013) Incidence and risk factors for hospital-acquired Clostridium difficile infection among inpatients in an orthopaedic tertiary care hospital. J Hosp Infect 83:146-149

27. Chitnis AS, Holzbauer SM, Belflower RM et al (2013) Epidemiology of community associated Clostridium difficile infection, 2009 through 2011. JAMA Intern Med 173:1359-1367

28. Kochan TJ, Somers MJ, Kaiser AM et al (2017) Intestinal calcium and bile salts facilitate germination of Clostridium difficile spores. PLoS Pathog 13:e1006443

29. Baktash A, Terveer EM, Zwittink RD et al (2018) Mechanistic insights in the success of fecal microbiota transplants for the treatment of Clostridium difficile infections. Front Microbiol 9:1242

30. Chiang JY (2009) Bile acids: regulation of synthesis. J Lipid Res 50:1955-1966

31. Francis MB, Allen CA, Shrestha R, Sorg JA (2013) Bile acid recognition by the Clostridium difficile germinant receptor, $\mathrm{CspC}$, is important for establishing infection. PLoS Pathog 9: e1003356

32. Thanissery R, Winston JA, Theriot CM (2017) Inhibition of spore germination, growth, and toxin activity of clinically relevant $\mathrm{C}$. difficile strains by gut microbiota derived secondary bile acids. Anaerobe 45:86-100

33. Allegretti JR, Kearney S, Li N et al (2016) Recurrent Clostridium difficile infection associates with distinct bile acid and microbiome profiles. Aliment Pharmacol Ther 43:1142-1153

34. Smits WK, Lyras D, Lacy DB et al (2016) Clostridium difficile infection. Nat Rev Dis Primers 2:16020

35. Androga GO, Hart J, Foster NF et al (2015) Infection with toxin A-negative, toxin B-negative, binary toxin-positive Clostridium difficile in a young patient with ulcerative colitis. J Clin Microbiol 53:3702-3704

36. Lyerly DM, Saum KE, MacDonald DK et al (1985) Effects of Clostridium difficile given intragastrically to animals. Infect Immun 47:349-352

37. Riegler M, Sedivy R, Pothoulakis C et al (1995) Clostridium difficile toxin $\mathrm{B}$ is more potent than toxin $\mathrm{A}$ in damaging human colonic epithelium in vitro. J Clin Invest 95:2004-2011

38. Savidge TC, Pan WH, Newman P et al (2003) Clostridium difficile toxin $\mathrm{B}$ is an inflammatory enterotoxin in human intestine. Gastroenterology 125:413-420

39. McDonald LC, Killgore GE, Thompson A et al (2005) An epidemic, toxin-gene variant strain of Clostridium difficile. $\mathrm{N}$ Engl J Med 353:2433-2441

40. Loo VG, Poirier L, Miller MA et al (2005) Apredominantly clonal multi-institutional outbreak of Clostridium difficile-associated diarrhea with high morbidity and mortality. N Engl J Med 353: 2442-2449

41. Rocha MFG, Maia MET, Bezerra LR et al (1997) Clostridium difficile toxin $\mathrm{A}$ induces the release of neutrophil chemotactic factors from rat peritoneal macrophages: role of interleukin- $1 \beta$, tumor necrosis factor alpha, and leukotrienes. Infect Imm 65: 2740-2746

42. Steiner TS, Flores CA, Pizarro TT, et al. (1997) Fecal lactoferrin, interleukin- $1 \beta$ and interleukin- 8 are elevated in patients with severe Clostridium difficile colitis. Clin. Diagn Lab Immun 4: 719-722

43. Vaishnavi C (2010) Clinical spectrum \& pathogenesis of Clostridium difficile associated diseases. Indian J Med Res 131: 487-499

44. McFarland LV, Mulligan ME, Kwok RY et al (1989) Nosocomial acquisition of Clostridium difficile infection. N Engl J Med 320: 204-210

45. Toshniwal R, Silva J Jr, Fekety R et al (1981) Studies on the epidemiology of colitis due to Clostridium difficile in hamsters. $\mathrm{J}$ Infect Dis 143:51-54

46. Samore MH, DeGirolami PC, Tlucko A et al (1994) Clostridium difficile colonization and diarrhea at a tertiary care hospital. Clin Infect Dis 18:181-187

47. McDonald LC, Gerding DN, Johnson S et al (2018) Clinical practice guidelines for Clostridium difficile infection in adults and children: 2017 update by the Infectious Diseases Society of America (IDSA) and Society for Healthcare Epidemiology of America (SHEA). Clin Infect Dis 66:e1-e48

48. Vincent JL, Rello J, Marshall J et al (2009) International study of the prevalence and outcomes of infection in intensive care units. $\mathrm{J}$ Am Med Assoc 302:2323-2329

49. Sidler JA, Battegay M, Tschudin-Sutter S et al (2014) Enterococci, Clostridium difficile and ESBL producing bacteria: epidemiology, clinical impact and prevention in ICU patients. Swiss Med Wkly 144:14009

50. McFarland LV (2008) Antibiotic-associated diarrhea: epidemiology, trends and treatment. Fut Microbiol 3:563-578

51. McFarland LV, Surawicz CM, Rubin M et al (1999) Recurrent Clostridium difficile disease: epidemiology and clinical characteristics. Infect Control Hosp Epidemiol 20:43-50

52. Fekety R, McFarland LV, Surawicz CM et al (1997) Recurrent Clostridium difficile diarrhea: characteristics of and risk factors for patients enrolled in a prospective, randomized, doubleblinded trial. Clin Infect Dis 24:324-333

53. Moore SC (2018) Clostridium difficile: more challenging than ever. Crit Care Nurs Clin North Am 30:41-53

54. Boyce JM, Havill NL, Otter JA et al (2008) Impact of hydrogen peroxide vapor room decontamination on Clostridium difficile environmental contamination and transmission in a healthcare setting. Infect Control Hosp Epidemiol 29:723-729

55. Bartlett JG (2010) Detection of Clostridium difficile infection. Infect Control Hosp Epidemiol 31:35-37

56. Crobach MJ, Dekkers OM, Wilcox MH et al (2009) Data review and recommendations for diagnosing Clostridium difficile infection (CDI). Clin Microbiol Infect 15:1053-1066

57. Su W, Mercer J, Van Hal SJ et al (2013) Clostridium difficile testing: have we got it right? J Clin Microbiol 51:377-378

58. Crobach MJT, Planche T, Eckert C, et al. (2016) European Society of Clinical Microbiology and Infectious Diseases: update of the diagnostic guidance document for Clostridium difficile infection Clinical Microbiology and Infection 22:S63-S81

59. American Academy of Pediatrics, Committee on Infectious Diseases. Red Book, 28th Edition. 2009 Report of the Committee on Infectious Diseases, pp. 263-265

60. Sethi AK, Al-Nassir WN, Nerandzic MM et al (2010) Persistence of skin contamination and environmental shedding of Clostridium difficile during and after treatment of $C$. difficile infection. Infect Control Hosp Epidemiol 31:21-27

61. Hookman P, Barkin JS (2009) Clostridium difficile associated infection, diarrhea, and colitis. W J Gastroenterol 15:1554-1580

62. Ananthakrishnan AN, Binion DG (2010) Impact of Clostridium difficile on inflammatory bowel disease. Expert Rev Gastroenterol Hepatol 4:589-600 
63. Goodhand JR, Alazawi W, Rampton DS (2011) Systematic review: Clostridium difficile and inflammatory bowel disease. Aliment Pharmacol Ther 33:428-441

64. Farooq PD, Urrunaga NH, Tang DM, von Rosenvinge EC (2015) Pseudomembranous colitis. Dis Mon 61:181-206

65. Paláu-Dávila L, Lara-Medrano R, Negreros-Osuna AA et al (2016) Efficacy of computed tomography for the prediction of colectomy and mortality in patients with Clostridium difficile infection. Ann Med Surg (Lond) 12:101-105

66. Debast SB, Bauer MP, Kuijper EJ, ESCMID (2014) European Society of Clinical Microbiology and Infectious Diseases: update of the treatment guidance document for Clostridium difficile infection. Clin Microbiol Infect 20:1-26

67. Johnson S, Louie TJ, Gerding DN et al (2014) Vancomycin, metronidazole, or tolevamer for Clostridium difficile infection: results from two multinational, randomized, controlled trials. Clin Infect Dis 59:345-354

68. Nelson RL, Suda KJ, Evans CT (2017) Antibiotic treatment for Clostridium difficile associated diarrhoea in adults. Cochrane Database Syst Rev 3:CD004610

69. Louie TJ, Miller MA, Mullane KM et al (2011) Fidaxomicin versus vancomycin for Clostridium difficile infection. N Engl J Med 364:422-431

70. Cornely OA, Crook DW, Esposito R et al (2012) Fidaxomicin versus vancomycin for infection with Clostridium difficile in Europe, Canada, and the USA: a double-blind, non-inferiority, randomised controlled trial. Lancet Infect Dis 12:281-289

71. Cornely OA, Miller MA, Louie TJ et al (2012) Treatment of first recurrence of Clostridium difficile infection: fidaxomicin versus vancomycin. Clin Infect Dis 55:S154-S161

72. Cornely OA, Nathwani D, Ivanescu C et al (2014) Clinical efficacy of fidaxomicin compared with vancomycin and metronidazole in Clostridium difficile infections: a meta-analysis and indirect treatment comparison. J Antimicrob Chemother 69:2892e900

73. Guery B, Menichetti F, Anttila VJ et al (2018) Extended-pulsed fidaxomicin versus vancomycin for Clostridium difficile infection in patients 60 years and older (EXTEND): a randomised, controlled, open-label, phase 3b/4 trial. Lancet Infect Dis 18:296-307

74. Czepiel J, Biesiada G, Dróżdż M et al (2018) The presence of IL-8 $+781 \mathrm{~T} / \mathrm{C}$ polymorphism is associated with the parameters of severe Clostridium difficile infection. Microb Pathog 114:281-285

75. Bricker E, Garg R, Nelson R et al (2007) Antibiotic treatment for Clostridium difficile-associated diarrhea in adults. Cochrane Database Syst Rev 3:CD004610

76. DuPont HL, Garey K, Caeiro JP et al (2008) New advances in Clostridium difficile infection: changing epidemiology, diagnosis, treatment and control. Curr Opin Infect Dis 21:500-507

77. Gerding DN, Johnson S (2011) Clostridium difficile infection in 2010: advances in pathogenesis, diagnosis and management of CDI. Nat Rev Gastroenterol Hepatol 8:67-68

78. $\mathrm{Hu}$ MY, Katchar K, Kyne L et al (2009) Prospective derivation and validation of a clinical prediction rule for recurrent Clostridium difficile infection. Gastroenterology 136:1206-1214

79. Mullane KM, Miller MA, Weiss K et al (2011) Efficacy of fidaxomicin versus vancomycin as therapy for Clostridium difficile infection in individuals taking concomitant antibiotics for other concurrent infections. Clin Infect Dis 53:440

80. Johnson S, Maziade PJ, McFarland LV et al (2012) Is primary prevention of Clostridium difficile infection possible with specific probiotics? Int J Infect Dis 16:786-792

81. Johnston BC, Ma SSY, Goldenberg JZ et al (2012) Probiotics for the prevention of Clostridium difficile-associated diarrhea: a systematic review and meta-analysis. Ann Intern Med 157:878-888
82. Goldenberg JZ, Ma SSY, Saxton JD et al (2013) Probiotics for the prevention of Clostridium difficile-associated diarrhea in adults and children (review). Cochrane Database Syst Rev 5:1-153

83. McFarland LV (2006) Meta-analysis of probiotics for the prevention of antibiotic associated diarrhea and the treatment of Clostridium difficile disease. Am J Gastroenterol 101:812-822

84. Evans CT, Johnson S (2015) Prevention of Clostridium difficile infection with probiotics. Clin Infect Dis 60:122-128

85. Mulligan ME, Miller SD, McFarland LV et al (1993) Elevated levels of serum immunoglobulins in asymptomatic carriers of Clostridium difficile. Clin Infect Dis 16:239-244

86. Goldberg EJ, Bhalodia S, Jacob S, et al. (2015) Clostridium difficile infection: A brief update on emerging therapies Am J Health-Syst Pharm 72:1007-1012

87. Siddiqui F, O'Connor JR, Nagaro K et al (2012) Vaccination with parenteral toxoid $\mathrm{B}$ protects hamsters against lethal challenge with toxin A-negative, toxin B-positive Clostridium difficile but does not prevent colonization. J Infect Dis 205:128-133

88. Kyne L, Warny M, Qamar A, Kelly CP (2001) Association between antibody response to toxin A and protection against recurrent Clostridium difficile diarrhoea. Lancet 357:189-193

89. Lowy I, Molrine DC, Leav BA et al (2010) Treatment with monoclonal antibodies against Clostridium difficile toxins. N Engl J Med 362:197-205

90. Wilcox MH, Gerding DN, Poxton IR et al (2017) Bezlotoxumab for prevention of recurrent Clostridium difficile infection. $\mathrm{N}$ Engl $\mathrm{J}$ Med 376:305-317

91. Zhang F, Luo W, Fan Z, Ji G (2012) Should we standardize the 1700 year old fecal microbiota transplantation? Am J Gastroenterol 107:755

92. Borody TJ, Warren EF, Leis SM et al (2004) Bacteriotherapy using fecal flora: toying with human motions. J Clin Gastroenterol 38:475-483

93. Eiseman B, Silen W, Bascom GS, Kauvar AJ (1958) Fecal enema as an adjunct in the treatment of pseudomembranous enterocolitis. Surgery 44:854-859

94. Schwan A, Sjolin S, Trottestam U, Aronsson B (1983) Relapsing Clostridium difficile enterocolitis cured by rectal infusion of homologous faeces. Lancet 2:845

95. Austin M, Mellow M, Tierney WM (2014) Fecal microbiota transplantation in the treatment of Clostridium difficile infections. Am J Med 127:479-483

96. Dutta SK, Girotra M, Garg S et al (2014) Efficacy of combined jejunal and colonic fecal microbiota transplantation for recurrent Clostridium difficile infection. Clin Gastroenterol Hepatol 12: $1572-1576$

97. Louie T, Cannon K, O'Grady H et al. (2015) Fecal microbiome transplantation (FMT) via oral fecal microbial capsules for recurrent Clostridium difficile infection (rCDI). https://idsa.confex. com/idsa/2013/ webprogram/Paper41627.Html

98. van Nood E, Vrieze A, Nieuwdorp M et al (2013) Duodenal infusion of donor feces for recurrent Clostridium difficile. N Engl J Med 368:407-415

99. Borody TJ, Khoruts A (2012) Fecal microbiota transplantation and emerging applications. Nature Rev Gastroenterol Hepatol 9: 88-96

100. Terveer EM, van Beurden YH, Goorhuis A et al (2017) How to: establish and run a stool bank. Clin Microbiol Infect 23:924e930

101. Bafeta A, Yavchitz A, Riveros C et al (2017) Methods and reporting studies assessing fecal microbiota transplantation: a systematic review. Ann Intern Med 167:34-39

Publisher's note Springer Nature remains neutral with regard to jurisdictional claims in published maps and institutional affiliations. 